This information is current as of April 26, 2023.

\title{
Visual Outcomes and Ophthalmic Aneurysms
}

T.K. Mattingly, D.M. Pelz and S.P. Lownie

AJNR Am J Neuroradiol 2015, 36 (4) E32 doi: https://doi.org/10.3174/ajnr.A4244

http://www.ajnr.org/content/36/4/E32 


\section{Visual Outcomes and Ophthalmic Aneurysms}

W e read with interest the article by Durst et al, "Vision Outcomes and Major Complications after Endovascular Coil Embolization of Ophthalmic Segment Aneurysms." ${ }^{1}$ On the basis of the results in their and other endovascular series compared with surgical series, the authors suggested that the likelihood of visual improvement in aneurysms treated by endovascular means may be similar to that obtained by surgical occlusion and that the risk of morbidity may be lower.

We are concerned about a number of methodologic shortcomings in this article, which may have led to erroneous interpretation by the authors. Only 7 of 22 cases were formally evaluated by a neuro-ophthalmologist. In only those 7 individuals had visual field defects and the presence of optic nerve atrophy been properly documented. This left 15 patients with no formal neuroophthalmologic evaluation of visual fields or funduscopy. We noted no formal evaluation of visual acuity. We also failed to understand the authors' inclusion of ophthalmoplegia or diplopia (observed in 6 cases), which is clinically inconsistent with optic nerve compression by ophthalmic aneurysms. The authors also included "visual pain" in their assessment of clinical findings. We have not previously seen this term recorded in well-documented series of visual outcomes after treatment of these lesions.

The average aneurysm size in the authors' series was only 9 $\mathrm{mm}$. It is widely recognized that aneurysms presenting with optic apparatus compression are typically $>1 \mathrm{~cm}$ in diameter, and most series exploring this feature comprise aneurysms of large or giant size. $^{2,3}$ Size also has a great bearing on treatment-related morbidity, yet it was hardly given due consideration by the authors. Therefore, we think the authors are premature in drawing any

http://dx.doi.org/10.3174/ajnr.A4244 conclusions about treatment from this series other than that endovascular treatment of small ophthalmic aneurysms is fairly safe.

We believe that there is no substitute for independent neuroophthalmologic evaluation in the pre- and postprocedural assessment of these challenging patients. ${ }^{4}$ The analysis of the results must include visual acuity, Goldman or Humphrey visual fields, and funduscopic evaluation for optic nerve atrophy and the presence of an afferent pupillary defect. Additionally, comparison of endovascular-versus-surgical series should include morphologic features of aneurysm size and location matched to accurate vision data. Only with comparable groups of patients and with aneurysms of a similar size should investigators be tempted to compare the results of endovascular and surgical occlusion.

\section{REFERENCES}

1. Durst CR, Starke RM, Gaughen J. Vision outcomes and major complications after endovascular coil embolization of ophthalmic segment aneurysms. AJNR Am J Neuroradiol 2014;35:2140-45

2. Day AL. Aneurysms of the ophthalmic segment. J Neurosurg 1990; 72:677-91

3. Date I, Asari S, Ohmoto T, et al. Cerebral aneurysms causing visual symptoms: their features and surgical outcome. Clin Neurol Neurosurg 1998;100:259-67

4. Mattingly T, Kole MK, Nicolle D, et al. Visual outcomes for surgical treatment of large and giant carotid ophthalmic segment aneurysms: a case series utilizing retrograde suction decompression (the "Dallas technique"). J Neurosurg 2013;118:937-46

T.K. Mattingly Neurosurgical Associates Richmond, Virginia D.M. Pelz

S.P. Lownie Departments of Medical Imaging and Clinical Neurological Sciences University Hospital/London Health Sciences Centre London, Ontario, Canada 